\title{
Análise de Qualidade de Serviço de VoIP em Redes com Controle de Congestionamento RED Adaptativo
}

\author{
Vitalio Alfonso Reguera, Evelio M. García Fernández, Walter Godoy Jr. e Félix F. Álvarez Paliza
}

\begin{abstract}
Resumo-Este artigo apresenta um novo método analítico baseado numa aproximação de ponto fixo para se estimar o impacto das técnicas de gerenciamento ativo de filas na qualidade de serviço em aplicações de voz sobre IP. Os resultados obtidos pelo método poposto foram validados usando técnicas de simulação de eventos discretos. Em todos os casos estudados se observa uma grande concordância entre os resultados analíticos e os resultados obtidos através de simulação.
\end{abstract}

Palavras-Chave-Gerenciamento ativo de filas, Voz sobre IP, Avaliação de desempenho, Internet.

Abstract-This article provides a new analytical method based on a fixed point approach to estimate the impact of active queue management schemes on the quality of service of voice over IP applications. The results obtained by the proposed method were validated using discret event simulation techniques. In all the studied cases it was observed a great deal of agreement between the analytical results and the results obtained through simulation.

Keywords-Active queue management, Voice over IP, Performance analyses, Internet.

\section{INTRODUÇÃO}

O gerenciamento ativo de filas (AQM: Active Queue Management) tem sido proposto como uma ferramenta para o controle de congestionamento na Internet [1]. Vários estudos têm-se dedicado nos últimos anos ao projeto e avaliação de algoritmos AQM [2]-[5]. Mecanismos de AQM atuam nos roteadores de rede enviando informação para as fontes geradoras de tráfego sobre o perigo de congestionamento iminente. Protocolos da camada de transporte da rede como o protocolo TCP (TCP: Transport Control Protocol) têm a capacidade de reagir a estes sinais diminuindo a taxa de envio de dados, evitando desta forma que se produza congestionamento. Em muitos casos o uso do gerenciamento ativo de filas ajuda a diminuir as alterações em parâmetros de rede tais como o atraso, variação do atraso (jitter) e perda de pacotes que são produzidas durante os períodos de congestionamento. $\mathrm{O}$ anterior se traduz num aumento da qualidade de serviço para as aplicações que são afetadas pela degradação destes parâmetros. Devido à complexa interação entre os mecanismos de controle

Vitalio Alfonso Reguera e Félix F. Álvarez Paliza, Departamento de Telecomunicações, Universidad Central de las Villas, Santa Clara, Cuba, E-mails: vitalio@uclv.edu.cu, fapaliza@uclv.edu.cu. , Evelio M. García Fernández, Departamento de Eng. Elétrica, Universidade Federal do Paraná, Curitiba, Brasil, E-mail: evelio@eletrica.ufpr.br. Walter Godoy Jr., Departamento de Eng. Elétrica, Universidade Tecnológica Federal do Paraná, E-mail: gogoy@utfpr.edu.br. Este trabalho foi parcialmente financiado pela CAPES (Capes/MES-Cuba 016/06). de rede e as aplicações no resulta fácil avaliar a influência que estes mecanismos exercem na percepção que os usuários têm sobre a qualidade de serviço oferecida. Na referência [5] esta avaliação foi feita através de simulação computacional verificando-se que a utilização de mecanismos de gerenciamento ativo de filas aumenta de maneira significativa a qualidade percebida pelos usuários na recepção de chamadas telefônicas transmitidas através de enlaces congestionados. Porém, modelos de simulação computacional não são capazes por si só de representar adequadamente toda a gama de interações possíveis entre fontes de tráfego e algoritmos de controle de congestionamento.

Neste trabalho é apresentado um procedimento analítico baseado em uma aproximação de ponto fixo para determinar o impacto de algoritmos AQM na qualidade de serviço de aplicações de voz sobre IP (VoIP: Voice over IP). O algoritmo de detecção adiantada aleatória (RED: Random Early Detection) é a proposta de AQM mais difundida e implementada na Internet [2]. Porém, a sua versão original tem mostrado ter um desempenho muito dependente dos parâmetros de configuração, o que produz resultados não desejados em alguns cenários de rede [6], [7]. A versão adaptativa de RED (ARED: Adaptive RED) soluciona este problema ajustando de forma dinâmica os parâmetros de controle do algoritmo.

O modelo proposto neste trabalho permite capturar a interação entre o algoritmo ARED e as fontes de tráfego, estimando os valores do atraso, variação do atraso e taxa de perda de pacotes. Uma vez obtidas as métricas de rede, estas são utilizadas como entradas do algoritmo computacional proposto em [8] que permite estimar o grau de satisfação experimentado pelos usuários de um sistema de voz sobre IP.

Uma descrição detalhada do método proposto aparece na próxima Seção. Na Seção III os resultados obtidos através do método analítico são contrapostos com os resultados obtidos através de simulação computacional. Por fim, na Seção IV aparecem na forma de conclusões, critérios sobre a validade e utilidade dos resultados obtidos.

\section{MÉTodos Analíticos}

O ponto de partida para o desenvolvimento do modelo matemático é um enlace congestionado onde opera o mecanismo de controle de congestionamento ARED. Considerando um roteador de Internet onde ingressam fluxos sensíveis à congestionamento (TCP) e outros que não o são (UDP: User Datagram Protocol), a interação entre estes fluxos e o 
mecanismo de gerenciamento ativo de filas do roteador pode ser representada como se ilustra na Figura 1. As fontes TCP regulam o tráfego que geram em dependência da taxa de perda de pacotes e do atraso de ida e volta (RTT: Round-Trip Time) que os pacotes experimentam. Pelo contrário, as fontes UDP são insensíveis ao grau de congestionamento que apresenta a rede.

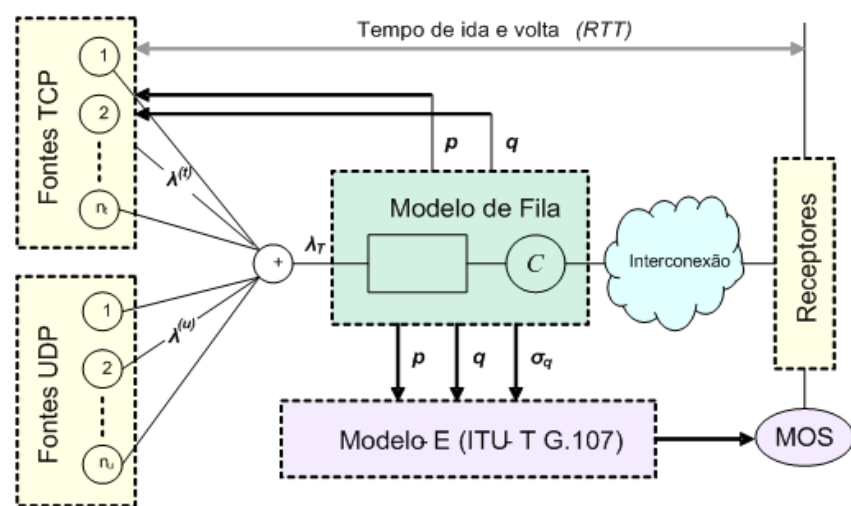

Fig. 1. Diagrama de Rede

Do ponto de vista da teoria de filas o roteador pode ser considerado como uma fila cuja disciplina de atendimento e tempo de serviço estarão determinados pelo mecanismo de gerenciamento ativo de filas atuante e pela capacidade do enlace de saída. Diferentemente da análise realizada em [9], neste trabalho tem-se em conta a realimentação que existe entre o sistema de fila e as fontes de tráfego, fazendo-as interagir de forma iterativa até encontrar a solução estável do sistema (ponto fixo). Esta idéia foi descrita pela primeira vez em [10] e posteriormente tem sido freqüentemente utilizada para modelar o comportamento de diferentes implementações de TCP [11], [12]. Partindo de um modelo preciso das fontes e da fila, o ponto fixo da iteração se corresponde com o ponto de operação da rede real. Uma vez obtidos os valores da taxa de perdas, do atraso médio na fila e da variação do atraso, estes parâmetros servem como entradas ao algoritmo computacional do Modelo-E [8] para estimar o valor médio de satisfação dos usuários (MOS: Mean Opinion Score) para as aplicações de voz.

\section{A. Aproximação de Ponto Fixo}

Sejam $\lambda_{i}^{(t)}$ e $\lambda_{i}^{(u)}$ a taxa de chegada gerada pela $i$-ésima fonte TCP e UDP respectivamente (ver Figura 1), então a taxa de chegada total, $\lambda_{T}$, é

$$
\lambda_{T}=\sum_{i=1}^{n_{t}} \lambda_{i}^{(t)}+\sum_{i=1}^{n_{u}} \lambda_{i}^{(u)}
$$

onde $n_{t}$ é o número de fontes TCP e $n_{u}$ é o número de fontes UDP. Sejam $q_{i}\left(\lambda_{T}\right)$ e $p_{i}\left(\lambda_{T}\right)$ o atraso médio na fila e a taxa de perda de pacotes calculada pelo modelo de fila e $t_{i}\left(q_{i}\left(\lambda_{T}\right), p_{i}\left(\lambda_{T}\right)\right)$ o throughput da $i$-ésima fonte TCP. Assumindo que a taxa de chagada das fontes UDP é constante e não é afetada pelo congestionamento da rede, então o ponto de operação pode ser encontrado de forma que satisfaça,

$$
\sum_{i=1}^{n_{t}} t_{i}\left(q_{i}\left(\lambda_{T}\right), p_{i}\left(\lambda_{T}\right)\right)+\sum_{i=1}^{n_{u}} \lambda_{i}^{(u)}=\lambda_{T}
$$

Note que a equação 2 é do tipo $f(x)=x$ onde $\lambda_{T}$ é um ponto fixo. Em dependência do modelo de fila utilizado nem sempre é possível encontrar uma expressão compacta para $q_{i}\left(\lambda_{T}\right)$ e $p_{i}\left(\lambda_{T}\right)$. Ademais, no modelo desenvolvido neste trabalho o ponto fixo no se comporta como um atrator; neste caso a solução da equação 2 pode ser encontrada utilizando-se métodos computacionais (Ex. usando-se o método de Brent).

\section{B. Modelo da Fonte}

Dado que o protocolo UDP não tem a capacidade de adaptar a taxa de transmissão às condições predominantes na rede, a taxa de chegada gerada pelas fontes associadas a este protocolo será considerada constante. Isto pode ser o caso de um codec de voz gerando pacotes a intervalos de tempo regulares com idêntico tamanho (Ex. G.711). Pelo contrário, as fontes suportadas no protocolo TCP adaptarão a taxa de transmissão em dependência do grau de congestionamento experimentado.

Nesta investigação se faz uso de um modelo amplamente utilizado para a estimação do desempenho de uma fonte TCP em estado estável proposto em [13]. Este modelo é aplicável às fontes TCP Reno, as quais são uma das implementações do protocolo de transporte mais difundidas na Internet [14]. Aqui o cálculo do desempenho é uma função da taxa de perda e do atraso de ida e volta experimentado pela conexão. Utilizando a mesma notação anterior e de acordo com [13], o desempenho de uma fonte TCP pode ser aproximado pela seguinte expressão,

$$
\begin{aligned}
t_{i}\left(q_{i}, p_{i}\right) & = \\
\min & \left(\begin{array}{l}
\frac{W_{m}}{R T T_{0}+q_{i}}, \\
\frac{1}{\left(R T T_{0}+q_{i}\right) \sqrt{\frac{2 b p_{i}}{3}}+T_{o} \min \left(1,3 \sqrt{\frac{3 b p_{i}}{8}}\right) p_{i}\left(1+32 p_{i}^{2}\right)}
\end{array}\right)
\end{aligned}
$$

onde $R T T_{0}$ é o atraso de ida e volta sem contar o atraso produzido pela fila, $W_{m}$ é o tamanho máximo de janela estabelecido pelo receptor (medido em segmentos), $T_{o}$ é o tempo de espera antes de realizar a primeira retransmissão e $b$ é o número de pacotes que são reconhecidos por ACK (tipicamente dois).

\section{Modelo de Fila ARED}

O modelo da fila vai estar determinado pelo mecanismo de gerenciamento ativo de fila utilizado e deve permitir estimar com adequada precisão a taxa de perda de pacotes, o atraso e a variação do atraso. Para isto faz-se uso dos principais resultados da teoria de filas adaptando-os à presença de mecanismos AQM. No caso do mecanismo ARED na fila do roteador a taxa de perda de pacotes estará condicionada pela função probabilidade de descarte estabelecida pelo algoritmo, segundo a expressão, 


$$
p_{R E D}\left(l_{q}\right)=\left\{\begin{array}{lr}
0, & l_{q}<\min _{t h} \\
1, & l_{q}>\max _{t h} \\
\max _{p}\left(\frac{l_{q}-\min _{t h}}{\max _{t h}-\min _{t h}}\right), & \text { demais valores }
\end{array}\right.
$$

onde os parâmetros $\min _{\text {th }}$ e $\max _{\text {th }}$ são os limitantes inferior e superior do tamanho médio da fila dentro dos quais a probabilidade de descarte tem um comportamento linear com inclinação positiva, $\max _{p}$ é o máximo valor de probabilidade nesse intervalo e $l_{q}$ é o tamanho médio da fila, atualizado a partir do valor instantâneo da fila, $I_{q}$, da seguinte forma

$$
l_{q} \longleftarrow\left(1-w_{q}\right) l_{q}+w_{q} I_{q}, \quad 0<w_{q}<1
$$

onde $w_{q}$ é o fator de ponderação que indica o peso que a amostra mais recente $\left(I_{q}\right)$ tem em relação à média acumulada $l_{q}$.

A probabilidade de ocupação da fila em estado estável pode ser obtida utilizando-se uma cadeia de Markov e aproximando a distribuição do processo de chegada e a do tempo de serviço por uma distribuição exponencial. Neste caso o processo se transforma em um processo de nascimento-morte. Aproximando o tamanho médio da fila pelo valor instantâneo do tamanho da fila, a probabilidade que no sistema existam $j$ pacotes no estado estável, $P_{j}^{A R E D}$, pode ser calculada como,

$$
P_{j}^{A R E D}=\frac{a^{j} \prod_{l=0}^{j-1}\left(1-p_{R E D}(l)\right)}{1+\sum_{i=1}^{K+1} a^{i} \prod_{l=0}^{i-1}\left(1-p_{R E D}(l)\right)}
$$

onde $a$ é o tráfego oferecido.

O algoritmo ARED pode ser configurado para descartar todos os pacotes com igual probabilidade (modo pacote) ou para descartar os pacotes em função do seu tamanho em bytes (modo byte). No primeiro caso a taxa de descarte de pacotes será a mesma para todas as fontes de tráfego e pode ser obtida como,

$$
p=\sum_{j=0}^{K+1} P_{j}^{A R E D} p_{R E D}(j)
$$

Quando a operação de ARED é no modo byte a taxa de perda experimentada pelas fontes dependerá do tamanho dos pacotes enviados. Neste caso a taxa de perda para a fonte $i$ é calculada como,

$$
p_{i}=\frac{\lambda_{T} h_{i}}{a} \sum_{j=0}^{K+1} P_{j}^{A R E D} p_{R E D}(j)
$$

onde $h_{i}$ é o tempo médio de serviço para a $i$-ésima fonte.

$\mathrm{O}$ atraso na fila experimentado pelos pacotes da fonte de tráfego $i$ pode ser calculado a partir da fórmula de Little [15] como,

$$
q=\frac{\sum_{j=0}^{K+1}(j-1) P_{j}^{A R E D}}{\lambda_{T}(1-p)}
$$

O jitter é estimado a partir do desvio padrão do atraso experimentado pelos pacotes. Seja $\sigma_{q}^{2}$ a variância do tempo de espera na fila do sistema, então considerando que um pacote que ingressa na fila deverá esperar por que cada um dos pacotes presentes no sistema quando da sua chegada sejam servidos, temos

$$
\sigma_{q}^{2}=Q \sigma_{h}^{2}+\sigma_{Q}^{2} h^{2}
$$

onde $Q$ é o número médio de pacotes no sistema, $\sigma_{Q}^{2}$ é a variância do número de pacotes no sistema, $h$ é o tempo médio de serviço e $\sigma_{h}^{2}$ é a variância do tempo médio de serviço.

A partir da equação 10 o jitter experimentado pela fonte de tráfego $i$ pode ser calculado como

$$
\sigma_{i}=\sqrt{\sigma_{q}^{2}+\sigma_{h_{i}}}
$$

onde $\sigma_{h_{i}}$ é a variância do tempo de serviço da fonte de tráfego $i$.

O ponto de equilíbrio da rede pode-se encontrar fazendo-se uso das equações 2, (7 ou 8) e 9. Uma vez determinado o ponto fixo, os valores obtidos através das equações (7 ou 8), 9 e 11 são usados para estimar o grau de satisfação dos usuários do sistema de VoIP.

Um procedimento similar ao descrito anteriormente poderia ser aplicado a outros algoritmos de gerenciamento ativo de filas, como por exemplo PI (PI: Proportional Integrator) e REM (REM: Random Exponential Marking), a partir de expressar as suas respectivas probabilidades de descarte em função do tamanho instantâneo da fila; o que leva a um desenvolvimento mais complexo que o apresentado aqui para o algoritmo ARED.

Em uma comunicação de voz através de Internet os pacotes viajam por múltiplos enlaces. Em dependência da rota estabelecida para a comunicação o congestionamento pode estar presente em mais de um enlace. O modelo anteriormente descrito para um enlace congestionado pode ser facilmente estendido a uma topologia com múltiplos enlaces. Neste caso a rede pode ser representada por um conjunto de filas em cascata onde o tráfego experimentado por um enlace ingressará na próxima fila misturando-se com o tráfego gerado por outro conjunto de fontes.

Aplicando para cada fila os procedimentos descritos nas subseções anteriores pode-se encontrar o ponto de operação do sistema como um todo no estado estável. A probabilidade de perdas, o tempo médio de espera e o jitter fim-a-fim nas filas considerando $m$ filas podem ser calculados, respectivamente como:

$$
\begin{gathered}
p=1-\prod_{\forall m}\left(1-p_{m}\right) \\
q=\sum_{\forall m} q_{m}
\end{gathered}
$$

e

$$
\sigma=\sqrt{\sum_{\forall m} \sigma_{m}^{2}}
$$




\section{Cálculo do MOS}

A escala MOS (MOS: Mean Opinion Score) [16] tem sido usada tradicionalmente para realizar medições subjetivas do grau de satisfação dos usuários em relação à percepção de comunicações de voz. A escala MOS tem valores de um até cinco, sendo que o maior valor corresponde à melhor qualidade na percepção. Uma vez que o MOS é um teste subjetivo difícil de ser realizado em situações práticas, outros testes objetivos têm sido desenvolvidos como, por exemplo, o Emodel, especificado na recomendação ITU G.107 [8]. O Emodel é um algoritmo computacional que incorpora fatores degenerativos presentes nas transmissões em redes e depois prediz a qualidade de voz que irá resultar dessas transmissões. A saída do E-model é um fator de qualidade, $R$, que é calculado como:

$$
R=R_{o}-I_{s}-I_{d}-I e_{e f f}+A
$$

onde $R_{O}$ representa a relação sinal-ruído, $I_{s}$ é uma combinação de todas as degradações que ocorrem mais ou menos simultaneamente com o sinal de voz, $I_{d}$ representa as degradações provocadas pelo atraso, $I e_{e f f}$ é um fator de degradação introduzido por equipamentos tais como codificadores e decodificadores de voz e $A$ é um fator de expectativa do usuário. A partir do cálculo do fator $R$ baseado na medição de parâmetros de rede, pode ser estimado o seu equivalente na escala MOS $\left(M O S_{C Q}\right)$ para transmissões de voz usando-se a seguinte equação

$$
M O S_{C Q}=1+0.035 R+R(R-60)(100-R) 7 \times 10^{-6}
$$

A equação 16 é válida para $0<R<100$. Para $R<0$, o valor de $M O S_{C Q}$ é fixado em 1 e para $R>100, M O S_{C Q}=$ 4,5 . Na Tabela I é mostrada a relação entre o fator $R$ do Emodel, o valor equivalente na escala MOS e a correspondente satisfação do usuário.

TABELA I

EQUIVALENCIA ENTRE O FATOR $R$ E A ESCALA MOS

\begin{tabular}{|l||r|c|}
\hline Satisfação dos Usuários & Fator $R$ & MOS \\
\hline \hline Muito Satisfeitos & 90 & 4,34 \\
\hline Satisfeitos & 80 & 4,03 \\
\hline Alguns insatisfeitos & 70 & 3,60 \\
\hline Muitos insatisfeitos & 60 & 3,10 \\
\hline Maioria insatisfeita & 50 & 2,58 \\
\hline
\end{tabular}

Com a utilização de parâmetros padrões [8] no cálculo, resultados que caracterizam uma alta qualidade são obtidos com um fator $R=93,2$. Tendo em consideração somente degradações da rede tais como atraso, jitter e perda de pacotes, a equação 15 pode ser rescrita como:

$$
R=93,2-I_{d}-I e_{e f f}
$$

Supondo que se trabalha com um perfeito cancelamento de eco na rede, o fator de degradação provocado pelo atraso se reduz a zero quando o atraso total em um sentido entre o lado transmissor e o lado receptor, $T_{a}$, é menor ou igual a $100 \mathrm{~ms}$. Para $T_{a}$ acima de $100 \mathrm{~ms}, I_{d}$ é calculado como:

$$
I_{d}=25\left\{\left(1+x^{6}\right)^{\frac{1}{6}}-3\left[1+\left(\frac{x}{3}\right)^{6}\right]^{\frac{1}{6}}+2\right\}
$$

onde

$$
x=\frac{\log \left(\frac{T_{a}}{100}\right)}{\log 2}
$$

O fator de degradação introduzido pelo uso de codecs de baixa taxa de bits, $I e$, encontra-se tabelado na recomendação ITU G.113 [17]. O valor do fator de degradação efetivo quando da operação dos codificadores sob condições de perda aleatória de pacotes é calculado como:

$$
I e_{e f f}=I e+(95-I e) \frac{p}{p+B_{p l}}
$$

onde o fator de robustez contra perda de pacotes, $B_{p l}$, é definido como sendo um parâmetro específico do codec na referência [17] e $p$ é calculada através da equação 7.

\section{REsultados E Discuss Ão}

Nesta seção são apresentados os resultados obtidos pelo procedimento proposto acima em um cenário de rede composto por um enlace de rede congestionado no qual foram misturadas três comunicações de voz com um número variável de fontes TCP. Os tempos de ida e volta para as conexões TCP variam entre 20 e 400 ms. O tamanho máximo dos segmentos TCP (MSS: Maximum Segment Size) foi ajustado em 1000 bytes que é aproximadamente a média dos valores de MSS comumente observados na Internet [18], [19]. O mecanismo de controle de congestionamento ARED foi ativado nos roteadores com uma capacidade máxima na fila cujo valor predeterminado foi de 120 pacotes. O comprimento desejado da fila foi igualado a 20 pacotes. Isto representa um atraso médio de aproximadamente $40 \mathrm{~ms}$ considerando um tamanho médio dos pacotes de 500 bytes e uma capacidade do enlace, $C$, de 2 Mbps. As comunicações de voz foram simuladas utilizandose fontes de tráfego com taxa de bit constante. Para isto se estabeleceram sessões UDP com carga útil de 92 bytes por pacote, 80 deles correspondentes às amostras de áudio e 12 ao cabeçalho RTP (RTP: Real-Time Transport Protocol). Os pacotes são enviados a uma taxa de 100 pacotes/s para simular um codificador G.711.

Na Figura 2 são mostrados os valores do registro médio de opinião obtido por meio do modelo matemático e através de simulação computacional utilizando-se o pacote de software network simulator (ns2) quando o algoritmo ARED é configurado em modo pacote. Para poder estabelecer um critério com relação aos benefícios do uso de AQM para o tráfego de voz também são apresentados os resultados da simulação para uma fila convencional FIFO (FIFO: First In First Out). Como se pode observar neste exemplo não há uma melhora significativa na qualidade de serviço quando a fila FIFO é substituída por uma fila ARED operando em modo pacote. Em ambos os casos o valor do MOS está abaixo do valor que 
expressa uma adequada satisfação dos usuários e diminui na medida em que o número de fontes de tráfego aumenta.

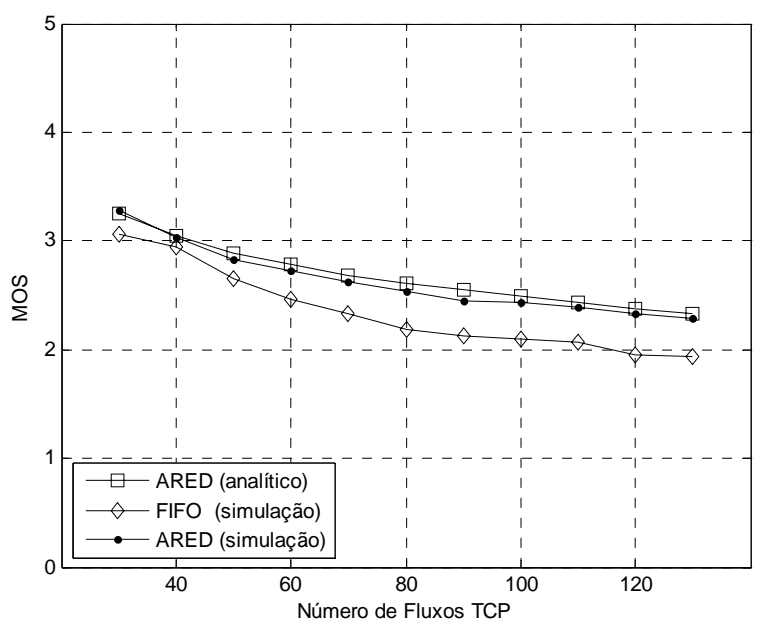

Fig. 2. Desempenho de ARED em modo pacote

Configurando o mecanismo ARED em modo byte foram obtidos os resultados apresentados na Figura 3. Agora o algoritmo AQM traz uma alta satisfação aos usuários das aplicações de voz, equivalente à do sistema telefônico tradicional (telefonia fixa). Isto se deve a que quando o algoritmo AQM trabalha em modo byte a probabilidade de perda aumenta para os pacotes de maior tamanho, beneficiando aplicações tais como as de VoIP que geram pacotes de pequeno tamanho. Estes resultados coincidem com outros estudos utilizando métodos empíricos apresentados em [5].

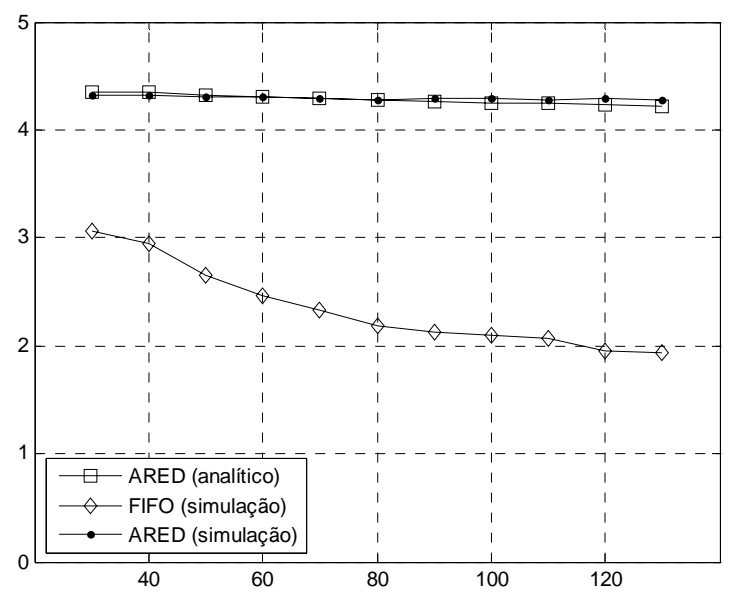

Fig. 3. Desempenho de ARED em modo byte

Como se pode apreciar tanto na Figura 2 como na Figura 3 os resultados obtidos a partir do modelo analítico são muito similares aos resultados da simulação. Isto também foi possível de ser evidenciado em uma ampla gama de experimentos não mostrados aqui por motivos de espaço.

\section{CONCLUSÕES}

Os exemplos numéricos descritos neste trabalho evidenciam que o método analítico proposto para a estimação da qualidade de serviço para as aplicações de voz fornece resultados similares aos resultados obtidos através de simulação computacional. Isto permite validar o seu uso para a avaliação do impacto dos mecanismos AQM na satisfação percebida pelos usuários finais de um sistema de voz sobre IP. Ademais, este método matemático pode servir como uma ferramenta para o projeto, dimensionamento e configuração dos sistemas de VoIP, permitindo estudar o desempenho dos mesmos e a sua interação com os mecanismos de controle de rede; encurtando os tempos necessários para isto em comparação ao método de simulação e com um custo extremadamente mais baixo em comparação aos testes com redes reais.

\section{REFERÊNCIAS}

[1] B. Braden, D. Clark, J. Crowcroft, et al, "Recommendations on Queue Management and Congestion Avoidance in the Internet.” RFC 2309, April 1998.

[2] S. Floyd and V. Jacobson, "Random early detection gateways for congestion avoidance", IEEE/ACM Trans. Networking, vol. 1, no. 4, pp. 397-413, August 1993.

[3] C. Hollot, V. Misra, D. Towsley, and W. Gong, "Analysis and design of controllers for AQM routers supporting TCP flows," IEEE Trans. Automat. Contr., vol. 47, no. 6, pp. 945-959, June 2002.

[4] M. Huggard, M. Robin, A. Bitorika, and C. McGoldrick, "Performance evaluation of fairness-oriented active queue management schemes”. In Proc. IEEE MASCOTS, pp. 105-112, 2004.

[5] V. A. Reguera, F. A. Paliza, E. M. G Fernández, W. Godoy Jr., "Voice over IP Quality of Service Using Active Queue Management" Proceedings of the VI International Telecommunications Symposium (ITS2006), Fortaleza-CE, Brasil, September de 2006.

[6] S. Floyd and E. Kohler, "Internet research needs better models." SIGCOMM Comput. Commun., Rev. 33(1):29-34, January 2003.

[7] M. May, J. Bolot, C. Diot, and B. Lyles, "Reasons not to deploy RED." In Proc. of 7th Int. Workshop on Quality of Service (IWQoS '99), London, June 1999.

[8] ITU-T Recommendation G.107, The E-model, a computational model for use in transmission planning, March 2005.

[9] Martin May, Thomas Bonald, and Jean-Chrysostome Bolot, "Analytic evaluation of RED performance," in Proceedings of IEEE Infocom, March 2000.

[10] C. Casetti and M. Meo. "A new approach to model the stationary behavior of TCP connections.” In Proc. of IEEE INFOCOM, pp. 367375, March 2000.

[11] C. Casetti and M. Meo. "An analytical framework for the performance evaluation of TCP Reno connections.” Computer Networks, 37(5): pp. 669-682, 2001.

[12] T. Bu and D. Towsley. "Fixed point approximations for TCP behavior in an AQM network.” In Proc. of ACM Sigmetrics, pp. 216-225, Jun. 2001.

[13] J. Padhye and V. Firoiu and D. Towsley and J. Krusoe, "Modeling TCP Throughput: A Simple Model and its Empirical Validation.” Proceedings of the ACM SIGCOMM '98, pp. 303-314, 1998.

[14] J. Padhye and S. Floyd, “On Inferring TCP Behavior.” ACM SIGCOMM, pp. 287-298, August 27-31, 2001.

[15] D. Gross, and C. M. Harris, "Fundamental of Queueing Theory”, Third Edition, John Wiley \& Sons, N.Y, 1998.

[16] ITU-T Recommendation P.800, Methods for subjective determination of transmission quality, August 1996.

[17] ITU-T Recommendation G.113 Appendix I, Provisional planning values for the equipment impairment factor Ie and packet-loss robustness factor Bpl, 2002.

[18] C. Fraleigh, ed al. "Packet-level traffic measurements from the Sprint IP backbone.” IEEE Network, 17(6), pp. 6-16, 2003.

[19] K. Thompson, G. Miller and R. Wilder, "Wide area Internet traffic patterns and characteristics,” IEEE Network, Nov 1997. 